Archives de sciences sociales des religions

116 | octobre - décembre 2001

Varia

\title{
Le Christianisme en Grande-Bretagne : débats et controverses autour d'une mort annoncée
}

Danièle Hervieu-Leger

\section{OpenEdition}

1 Journals

Édition électronique

URL : http://journals.openedition.org/assr/543

DOI : $10.4000 /$ assr.543

ISSN : $1777-5825$

Éditeur

Éditions de l'EHESS

Édition imprimée

Date de publication : 2 octobre 2001

Pagination : 31-40

ISBN : 2-222-96712-0

ISSN : 0335-5985

Référence électronique

Danièle Hervieu-Leger, «Le Christianisme en Grande-Bretagne : débats et controverses autour d'une mort annoncée ", Archives de sciences sociales des religions [En ligne], 116 | octobre - décembre 2001, mis en ligne le 17 octobre 2005, consulté le 30 avril 2019. URL : http://journals.openedition.org/ assr/543 ; DOl : 10.4000/assr.543 


\section{LE CHRISTIANISME EN GRANDE-BRETAGNE : DÉBATS ET CONTROVERSES AUTOUR D'UNE MORT ANNONCÉE}

\footnotetext{
À propos de :

BROWN (Callum G.), The Death of Christian Britain, Understanding Secularization 1800-2000, LondresNew York, Routledge, 2001, 198 p. (bibliogr., index).

BRUCE (Steve), Religion in the Modern World. From Cathedrals to Cults. Londres, Oxford University Press, 1996a, 234 p. (bibliogr., index).

BRUCE (Steve), «Religion in Britain at the Close of the 20th Century: A Challenge to the Silver Lining Perspective », Journal of Contemporary Religion, vol. 11, no 3. 1996b.

BRUCE (Steve), Choice and Religion. A Critique of Rational Choice, Londres, Oxford University Press, 1999, 186 p. (bibliogr., index, appendix).

BRUCE (Steve), "Christianity in Britain, R.I.P », Sociology of Religion, vol. 62, $\mathrm{n}^{\circ}$ 2, 2001, pp. 191-203.

DAVIE (Grace), Religion in Modern Europe. A Memory Mutates, Oxford, Oxford University Press, 2000a, 194 p. (bibliogr., index).

DAVIE (Grace), «Religion in Modern Britain: Changing Sociological Assumptions ", Sociology, vol. $34, \mathrm{n}^{\circ} 1,2000 \mathrm{~b}$, pp. 113-128.

GILL (Robin), HADAWAY (C. Kirk), MARLER (Penny Long), "Is Religious Belief Declining in Britain? », Journal for the Scientific Study of Religion, 37, 1998, pp. 507-516.

HORNSBY-SMITH (Michael), ed., Catholics in England, 1950-2000. Historical and Sociological Perspectives. Londres, Cassel, 1999, 306 p. (bibliogr., index).
} 
JENKINS (Timothy), Religion in English Everyday Life. An Ethnographic Approach, New York-Londres, Berghahn Books, 1999, 237 p. (bibliogr., index).

NYE (Malory), Multiculturalism and Minority Religion in Britain. Krishna Consciousness, Religious Freedom and the Politics of Location, Richmond (Surrey, G.-B.), Curzon Press, 2001, 305 p. (bibliogr., index).

Ouvrages et articles plus anciens et/ou déjà recensés dans les ASSR, et cités dans le cours de cette note:

BRUCE (Steve), ed., Religion and Modernization. Sociologists and Historians Debate the Secularization Thesis, Londres, Clarendon Press-Oxford, 1992, 210 p. (bibliogr., index).

BRUCE (Steve), Religion in Modern Britain, Oxford, Oxford University Press, 1995.

DAVIE (Grace), Religion in Britain Since 1945. Believing Without Belonging, Oxford, Blackwell, 1994, 211 p. (bibliogr., index.) (traduction française, 1996, La religion des britanniques. De 1945 à nos jours. Genève, Labor et Fides, 249 p.).

HORNSBY-SMITH (Michael), Roman Catholics in England, Cambridge, Cambridge University Press, 1987, 217 p. (bibliogr., index, appendix).

HORNSBY-SMITH (Michael), The Changing Parish. A Study of Parishes, Priests, and Parishioners after Vatican II, Londres-New York, Routledge, 1989, 211 p. (bibliogr., index, appendix).

HORNSBY-SMITH (Michael), Roman Catholic Beliefs in England. Customary Catholicism and Transformations of Religious Authority, Cambridge, Cambridge University Press, 1991, 231 p. (bibliogr., index, appendix).

HORNSBY-SMITH (Michael), «Believing Without Belonging? The Case of the Roman Catholics in England », in Bryan WILSON (ed), Religion: Contemporary Issues, Londres, Bellew Publishing, 1992.

JENKINS (Timothy), « Two Sociological Approaches to Religion in Modern Britain », Religion n ${ }^{\circ} 26,1996$, pp. 331-342.

MARTIN (David), «The Secularization Issue: Prospect and Retroprospect ». British Journal of Sociology, no 42, 1991, pp. 465-474.

MODOOD (Tariq), ed, Church, State and Religious Minorities, Londres, Policy Studies Institute, 1997.

PICKERING (W.S.F.), Anglo-Catholicism. A study in Religious Ambiguity. Londres-New York, Routledge, 1989, 269 p. 
Depuis une dizaine d'années, un ensemble impressionnant d'ouvrages et d'articles portant sur l'état de la vie religieuse, et plus spécifiquement du christianisme, en Grande-Bretagne ont été publiés : cette production nourrit le débat vigoureux (et parfois fort polémique) engagé entre nos collègues britanniques - sociologues, anthropologues et historiens - quant à la validité des approches classiques de la sécularisation appliquées au cas britannique. La présente note de lecture n'a pas pour objectif de recenser de façon exhaustive l'ensemble des travaux qui s'inscrivent dans ce débat, mais plutôt de saisir quelques articulations d'une discussion qui concerne, au-delà des dossiers historiques et empiriques anglais, l'ensemble des sociétés occidentales (européennes) auxquelles le paradigme de la perte religieuse des sociétés modernes est supposé s'appliquer de façon privilégiée, et probablement exclusive. On rappellera, pour mémoire, que Bryan Wilson, professeur à All Souls College (Oxford), fut l'un des théoriciens privilégiés de cette problématique de la perte, et que la synthèse brillante qu'il en offrit dès 1966 est devenue un «classique» de la sociologie des religions, au-dedans et au-dehors de la Grande-Bretagne ${ }^{1}$. Un peu plus de dix ans plus tard, David Martin, professeur à la London School of Economics, proposait une théorie générale de la sécularisation qui marquait le caractère non linéaire du processus d'éviction de la religion et insistait notamment sur les «filtres culturels » qui lui donnent, selon les contextes sociaux et culturels, une allure profondément différente ${ }^{2}$. Il n'est pas inutile de signaler d'emblée à quel point la référence à ces débats déjà un peu anciens demeure un point nodal dans les controverses présentes de la sociologie britannique des religions.

Le débat en cours prit naissance au tournant des années quatre-vingt, lorsque ce paradigme qui avait gouverné la recherche en sciences sociales des religions pendant plus de trente ans fut confronté à la découverte du foisonnement des Nouveaux mouvements religieux, révélateur supposé de la vitalité religieuse des sociétés dites «sécularisées ». L'urgence d'une "révision » du modèle fondateur sembla alors s'imposer, en même temps que fleurissaient les approches nouvelles $\mathrm{du}$ " retour du religieux », des « résurgences du sacré » ou de la "revanche de Dieu » dans les sociétés modernes. Il apparut assez vite que celles-ci ne pouvaient nourrir un véritable renouvellement des approches sociologiques classiques que dans la mesure où, renonçant à tordre le bâton théorique de la sécularisation dans l'autre sens, elles conduisaient à intégrer, comme les deux faces d'un même processus, l'analyse du fait mesurable de la disqualification des formes instituées de la religion et l'interprétation du déploiement de la croyance dans les sociétés modernes. On observa cependant une forte polarisation des points de vue, opposant « secularisationists" d'une part, et « revisionists» d'autre part (cf. Bruce, ed. 1992). En Grande-Bretagne, une floraison d'articles déclinèrent (et continuent de décliner) les termes évolutifs de ce débat (parmi ceux-ci : Martin, 1991 ; Jenkins, 1996 ; Bruce, 1996b. ; Gill, Hadaway and Marler, 1998 ; Davie, 2000 etc.). Confrontés au développement des recherches portant sur les formes nouvelles du croire dans les sociétés sécularisées, les tenants intransigeants de la problématique de la perte religieuse maintenaient, de leur côté, leur refus d'une reformulation quelconque du modèle classique de la sécularisation en mettant en avant la dispropor-

\footnotetext{
${ }^{1}$ Bryan Wilson, Religion in Secular Society, Londres, CA Watts, 1966.

2 David Martin, A General Theory of Secularization, New York, Harper and Row, 1978.
} 
tion - quantitative aussi bien que qualitative - entre le fait avéré constitué par l'affaissement de la religion instituée et les recompositions supposées des croyances contemporaines.

Parmi ceux-ci, Steve Bruce, professeur à l'Université d'Aberdeen, s'engagea très tôt, avec une fervente détermination, dans la défense de la théorie classique. Tout en soulignant - dans l'introduction de l'ouvrage collectif de 1992, co-signée avec notre regretté collègue Roy Wallis peu de temps avant la mort de celui-ci - la nécessité d'arracher cette théorie à toute vision téléologique d'un sens inéluctable (et peut-être désirable) de l'histoire de la modernité, il affirmait que le "modèle orthodoxe », définitivement étayé par un vaste ensemble de données empiriques, résistait parfaitement bien à toutes les critiques. Y compris celles de ceux qui, tels R. Stark et W.S. Bainbridge par exemple, lui opposaient l'irréductibilité des besoins psychologiques et individuels auxquels répond la religion, besoins qui trouvent - en situation d'évidement des grandes religions instituées - des formes compensatoires de satisfaction susceptibles de limiter, de l'intérieur, le processus même de la sécularisation ${ }^{3}$. La pugnacité intellectuelle de Steve Bruce a trouvé, depuis, à se déployer sur deux fronts principaux.

Le premier est celui de son débat avec notre collègue de l'Université d'Exeter, Grace Davie, auteur en 1994 d'un livre remarqué sur la religion des Britanniques depuis 1945, livre traduit en français en 1996. Jean-Paul Willaime a donné dans les Archives de Sciences Sociales des Religions (cf. Arch. 88.22), une recension détaillée - à laquelle on se rapportera - de cet ouvrage important, auquel son heureux sous-titre («Believing without belonging ») a valu de devenir l'emblême d'un ensemble de travaux portant sur les formes nouvelles de la religiosité dans les sociétés occidentales contemporaines. L'ouvrage de S. Bruce, paru en 1995, et dont le titre fait directement écho à celui de G. Davie, cristallise d'autant plus nettement l'enjeu de la discussion entre les deux auteurs que l'un et l'autre font appel aux mêmes données empiriques, dont ils tirent des interprétations divergentes. Pour G. Davie, l'effondrement mesurable des pratiques religieuses - tout particulièrement au sein de l'Église d'Angleterre - correspond moins à un rétrécissement des croyances qu'à un vaste processus de réorganisation des significations, inséparable de la disqualification des dispositifs d'accréditation officielle du croire. Cette disqualification produit, en même temps qu'elle mine les modalités traditionnelles de l'affiliation religieuse, une recomposition de la mémoire partagée, elle-même favorable à des réemplois inédits d'un ensemble de références symboliques, largement déconnectées de la syntaxe du croire prescrite par l'institution, mais qui demeurent opératoires, en particulier à l'occasion de la célébration des grands moments de la vie, ou dans des circonstances collectives exceptionnelles, comme celles d'un deuil public d'ampleur nationale. G. Davie offre, à propos des manifestations collectives du deuil mises en oeuvre lors de la tragédie du stade de Hillsborough en avril 1989, ou des différentes célébrations qui ont entouré le décès de la princesse Diana, une démonstration éblouissante de ces jeux complexes de l'invention rituelle et de la mobilisation mémorielle. La thèse de G. Davie, que l'ouvrage paru en 2000a approfondit en l'élargissant à l'espace européen, ne méconnaît en rien que les sociétés modernes occidentales sont bien des sociétés "sorties de la religion », dans les-

\footnotetext{
${ }^{3}$ R. StARK and W.S. BAInBridge, The Future of Religion. Secularization, Revival and Cult Formation. Berkeley, University of California Press, 1985.
} 
quelles les institutions religieuses ont perdu la capacité de définir, pour la société et même pour leurs membres, des croyances, des normes morales, des pratiques et des règles de comportement obligatoires. Mais elle s'efforce, avec une grande finesse, de combiner cette analyse classique avec la prise en compte du travail d'imprégnation culturelle que le christianisme a réalisé dans la longue durée de ces sociétés : un enracinement qui n'organise plus un univers commun du croire, mais qui alimente une "mémoire déléguée » (a vicarious memory), précaire certes, autant que sévèrement concurrencée à l'heure de la communication globalisée, mais qui demeure néanmoins un capital symbolique toujours actif, disponible (et même instrumentalisable) pour des utilisations plus ou moins ponctuelles, dans lesquelles s'inscrit néanmoins la continuité, paradoxale car fugace, d'une tradition.

Mobilisant l'artillerie lourde des données statistiques qui mettent en évidence l'effondrement des pratiques, S. Bruce balaye ces approches subtiles, auxquelles il reproche, de façon plus ou moins explicite (souvent très explicite!), de tenter de sauver, contre toute évidence, quelque chose d'un univers chrétien en déshérence. Un article tout récent, paru en 2001 dans la revue américaine Sociology of Religion, enfonce le clou en posant, dès son titre, l'acte de décès du christianisme en Grande-Bretagne : Christianity in Britain, R.I.P. Requiescat in pace! Qu'il repose en paix ! On pourra repérer, dans cette réaffirmation sans nuance de la thèse reprise de livre en livre, en 1995, en 1996b (sous une forme plus systématiquement théorique) et en 1999, un goût certain pour la provocation. Cette dernière considération ne doit cependant pas conduire à dévaluer le sérieux et la cohérence de la position défendue par S. Bruce, même si on ne la partage pas. Bruce fait partie de ceux qui considèrent que la consistance d'une " religion » se mesure avant tout à sa capacité d'organiser des pratiques et d'ordonner des comportements. Les tours, détours et recompositions de la croyance l'intéressent moins, de ce point de vue finalement très durkheimien, que ce lien structurant qui a nourri la puissance civilisationnelle et politique du christianisme pendant plusieurs siècles et qui se trouve aujourd'hui définitivement défait. Sur ce terrain, le constat ne souffre pas, selon lui, le moindre doute : l'effondrement du christianisme institutionnel atteint, en Grande-Bretagne (et ailleurs en Europe), un point qui justifie de s'interroger sur sa capacité, à court terme, de se reproduire comme religion au sein de ces sociétés. Bruce admet, par exemple, que les projections statistiques à l'horizon des années 2030 justifient l'hypothèse selon laquelle les principales dénominations chrétiennes auront alors disparu de la scène britannique...

Pour bien saisir l'enjeu de la position radicale tenue par S. Bruce, il faut réaliser qu'il ne se contente pas ici de débattre avec G. Davie, mais qu'il engage, en même temps, une vive polémique avec un certain nombre des représentants de la théorie des choix rationnels - au premier rang desquels le sociologue américain Rodney Stark - dont les vues, concernant le traitement des données statistiques portant sur la religion en Grande-Bretagne dans les cent-cinquante dernières années, défient directement l'hypothèse du "déclin religieux », associé par les « secularisationists » à la trajectoire de la modernisation. La problématique de la modernité religieuse développée par Grace Davie atteint la thèse de la sécularisation par l'aval, en discutant (sans récuser celle-ci comme telle) le caractère exagérément abrupt d'une perspective qui infère du constat de la perte institutionnelle de la religion la disparition pure et simple de toute forme consistante de religiosité dans les sociétés européennes. La problématique de la non-sécularisation tenue par 
Stark défie la thèse de la perte par l'amont : en remettant en question, à partir d'une relecture des données empiriques, l'idée que les sociétés du passé étaient plus imprégnées de religiosité et plus fermement gouvernées par la religion institutionnelle que ne le sont nos sociétés. Cette dernière approche est beaucoup plus meurtrière pour le «modèle orthodoxe » que ne l'est la précédente, puisqu'elle ne souffre aucune composition possible avec le schéma d'évolution que postule la théorie de la sécularisation : une évolution qui conduirait, depuis un siècle et demi, d'une société plus religieuse à une société moins religieuse. Dans un article paru en $1994^{4}$, R. Stark et L. Iannaconne, considérant les chiffres de l'affiliation religieuse et de la pratique régulière, mettaient sérieusement en question l'idée que la Grande-Bretagne fut beaucoup plus religieuse en 1800 qu'elle ne l'est aujourd'hui. Après tout, le "bricolage » des croyances, cher aux sociologues de la modernité religieuse, avait cours dans le passé tout autant qu'aujourd'hui. Les individus en prenaient et en laissaient avec les croyances orthodoxes autant qu'avec les normes morales prescrites par les Églises, et ne manifestaient certainement pas plus de respect à l'égard du clergé qu'ils ne lui en accordent aujourd'hui. La thèse de la sécularisation repose, selon les deux auteurs, sur le mythe d'un Âge d'Or de la chrétienté à tout le moins problématique : mettre à mal ce mythe revient, ipso facto, à déboulonner le prestige idéologique du paradigme fondateur de la sociologie des religions... L'ouvrage publié par Bruce en 1999, comme son article de 2001, ne se contente pas de réaffirmer l'évidence de la trajectoire du déclin, à partir de données réexaminées et enrichies. Il entend porter le fer au cœur de la construction théorique qui fonde la réinterprétation relativisante de ces données, en soulignant la nécessité de distinguer entre la religion comme culture et la religion des individus. Une telle distinction permet, par exemple, de ne pas confondre les accommodements de fait pris par les individus avec les croyances et pratiques officielles à l'intérieur d'un monde qui demeurait globalement gouverné par des références et normes religieuses, et les petits récits que les croyants modernes revendiquent de construire eux-mêmes, au nom des droits imprescriptibles de la subjectivité, dans un monde où la référence partagée à un cosmos religieux a cessé de faire sens. «The crucial point, and the conclusion toward which I will argue - écrit-il dans son article de 2001 - is that the superficial similarity of the present and the distant past in terms of the proportions of committed Christians disguises the far greater difference in the extent to which the background culture of our society is informed by religious beliefs and sustained by frequent low intensity affirmations of those beliefs ».

La critique ici portée à l'individualisme méthodologique radical qui sous-tend l'approche des tenants de la théorie des choix rationnels en matière religieuse est transparente, et, à notre sens, pleinement justifiée. Mais on peut se demander si cette récusation des présupposés méthodologiques de ceux qui considèrent que la Grande-Bretagne n'a jamais été aussi chrétienne que le postulent les «secularisationists » n'affaiblit pas, du même coup, la critique apportée par S. Bruce lui-même à la "silver lining perspective » qu'il prête à G. Davie. Admettons en effet (et on suivra volontiers S. Bruce sur ce terrain) que les données concernant la participation religieuse institutionnelle des individus ne livrent pas, à elles-seules, la clé de

4 «A Supply-Side reinterpretation of the 'Secularization' of Europe », Journal for the Scientific Study of Religion, no 33, 1994, pp. 230-252. 
l'état religieux de la société concernée. L'argument opposé à R. Stark se retourne alors en faveur de la thèse avancée par G. Davie. Du petit nombre des chrétiens pratiquants en leur sein, on ne peut inférer, nous dit Bruce, que les sociétés du passé n'étaient pas des sociétés gouvernées par la religion. De l'affaissement dramatique de cette participation aujourd'hui, on ne saurait davantage conclure, si on le suit jusqu'au bout, à l'expulsion définitive de la culture chrétienne dans une Grande-Bretagne sécularisée «sans reste ». À tout le moins, les formes, persistances et remodelages éventuels de cette imprégnation culturelle demandent, comme y appelle G. Davie, à être examinés de près.

Laissons ici les échanges d'arguments entre tenants et détracteurs (ou critiques) du "modèle orthodoxe» de la sécularisation dont S. Bruce s'est fait le héraut : l'intérêt intellectuel de ces débats internes à la sociologie britannique et anglo-saxonne (dont on peut déplorer qu'ils s'élargissent trop peu - G. Davie constituant ici (avec notre collègue de l'Université de Warwick, J. Beckford) une exception - à des discussions poursuivies sur ces thèmes dans d'autres univers linguistiques, français, italiens, allemands ou autres) est de poser, d'une façon qui mérite d'être systématiquement approfondie (non seulement dans le cas anglais, mais s'agissant de l'ensemble des sociétés d'Europe), la question des différents niveaux de description auxquels la trajectoire de la sécularisation peut être saisie.

- Un premier niveau - le mieux couvert assurément par les études empiriques disponibles - est celui de l'état de la religion institutionnelle, autrement dit celui des croyances et pratiques administrées par les grandes Églises. De ce point de vue, toutes les données viennent confirmer l'hypothèse classique d'une homogénéisation spirituelle de la Grande-Bretagne et de l'ensemble de l'Europe, sous le signe de la perte des grandes religions et de l'individualisation du croire. Toute la démonstration de $\mathrm{S}$. Bruce se déploie dans ce registre et elle le conduit (Bruce, 1999, chap. 7, « Liberal Religion ») à souligner justement la fragilité des formes de sociabilité religieuse que cette religion de l'individu, dans laquelle l'autonomie des sujets croyants l'emporte sur le pouvoir de la communauté, est susceptible de susciter, et ceci à l'intérieur même des grandes Églises. Toute la question est de savoir jusqu'à quel point cette socialisation précaire et instable implique un évidement inéluctable de la croyance. Pour S. Bruce, la conclusion est claire : « choice undermines faith ». Cette formule frappante résume parfaitement le propos général de l'ouvrage. « While the mutation of religion from church and sect into denomination and cult types may be seen like successful adaptation to increasing cultural diversity and individual autonomy, it is unlikely to retard secularization» (p. 159). « From cathedrals to cults » (Bruce, 1996a), le parcours est, indiscutablement selon Bruce, celui d'une irrémédiable perte.

Tout le problème est de savoir à quels facteurs économiques, sociaux, politiques et culturels il revient, dans la longue durée, d'imputer cette perte. De ce point de vue, les travaux de S. Bruce ne témoignent pas d'une grande originalité : ils reprennent, sous une forme très classique, la perspective wilsonienne selon laquelle le déploiement de la rationalité scientifique et technique, la fragmentation des sociétés et de la vie sociale liée au développement économique et à l'urbanisation, et le développement des grandes structures bureaucratiques à l'échelle nationale et internationale ont sonné, depuis le début du XIX ${ }^{\mathrm{e}}$ siècle au moins, le glas d'un style de vie communautaire et rural inséparablement associé à l'organisation chrétienne - sociale, axiologique et symbolique - du monde (Bruce, 1996a, chap. 3). 
Ce schéma est vigoureusement mis en question par l'étude de l'historien Callum G. Brown, co-directeur du Scottish Oral History Centre de l'Université de Strathclyde, étude qui montre au contraire - en s'éloignant des statistiques de pratique, mais en s'appuyant sur un vaste corpus documentaire, fait de romans, journaux, tracts, journaux intimes, autobiographies et notices nécrologiques l'extraordinaire prégnance d'une culture chrétienne qui continua d'informer les constructions narratives de soi-même, la moralité et le langage commun, des années 1800 jusqu'aux années 1960 (Brown, 2001). La césure qui marque véritablement la fin d'un monde religieux - The Death of Christian Britain - n'est pas la révolution technique, industrielle et urbaine du $\mathrm{XIX}^{\mathrm{e}}$ et de la première moitié du $\mathrm{XX}^{\mathrm{e}}$ siècle. C'est celle de la révolution des années 1960 : révolution morale et culturelle dont la clé est certainement la mutation de la famille et la redéfinition radicale des rapports de genres qui lui correspond. Ce déplacement de l'approche - de l'enregistrement des comportements religieux à l'étude des mentalités collectives et des moeurs - ne remet probablement pas autant en question que le suggère C. G. Brown les études classiques du déclin de la religion institutionnelle. Et ceci d'autant moins que son analyse du tournant culturel des années soixante demeure, sur le fonds, assez impressionniste. Mais le changement de point de vue qu'il opère invite à entrer dans un autre niveau d'analyse de la sécularisation.

- Ce second niveau est celui de la mise en perspective du travail civilisationnel des grandes traditions religieuses, en Grande-Bretagne comme dans les différents pays d'Europe : une mise en perspective qui fait ressortir, à rebours de la tendance précédente à l'homogénéisation sécularisée, la remarquable préservation à l'intérieur d'un fonds chrétien commun - de cultures religieuses nettement différenciées. Les enquêtes menées depuis des années par Michael Hornsby-Smith, professeur à l'Université du Surrey, sur les catholiques en Grande-Bretagne (Hornsby-Smith, 1987 [cf. Arch. 82.47], 1989, 1991, 1999), ou encore l'étude réalisée en 1989 par W.S.F. Pickering sur l'Anglo-Catholicisme illustrent bien, non seulement les transformations et changements, mais également les persistances de ces différenciations, culturelles autant que religieuses, internes au christianisme britannique. Ces différentes cultures chrétiennes sont aujourd'hui largement déboîtées des dispositifs confessionnels au sein desquels elles ont pris corps historiquement, mais elles demeurent présentes, de façon invisible ou semi-visible, dans des valeurs, pratiques et comportements qui ne revendiquent plus nécessairement une référence religieuse explicite. Sur ce second terrain, les analyses de G. Davie sur les remaniements et les nouvelles formes de mobilisation de la mémoire religieuse, sur fonds de perte des appartenances institutionnelles, se révèlent particulièrement précieuses. Car elles mettent en évidence, en même temps que la logique générale de ces processus, la manière dont ceux-ci s'incrivent dans un terreau culturel spécifique : celui, en l'occurrence, d'un anglicanisme dont la force dominante d'imprégnation culturelle est loin d'être entièrement dissipée ${ }^{5}$.

C'est également sur ce terrain que l'on mesure l'intérêt de multiplier, à côté des grandes enquêtes empiriques sur les croyances et les pratiques, des études ethnologiques du vécu religieux et spirituel quotidien des individus et des groupes, à

5 Cette approche appelle évidemment les mises en perspectives comparatives à l'échelle européenne auxquelles introduit précisément l'ouvrage paru en 2000 : Religion in Modern Europe. A Memory Mutates. 
l'échelle locale : c'est à l'échelle microsociologique en effet que la profondeur de ce travail d'imprégnation peut être saisie. C'est ce travail d'investigation au ras de la réalité ordinaire qui confère son intérêt, par exemple, à l'ouvrage de Timothy Jenkins, paru en 1999. Explorant les traits minuscules de la vie quotidienne d'une paroisse rurale (St. Mary's, Comberton, non loin de Cambridge), les enjeux sociaux, politiques et relationnels de l'organisation annuelle d'une procession (The Whit Walk) dans les faubourgs de Bristol, ou encore les thématiques associées aux croyances de genre spirites, alchimistes, astrologiques etc. présentées dans un livre de Michael Perry (Deliverance) publié en 1987 sous l'égide du Christian Exorcism Study Group, l'auteur s'efforce de développer une approche ethnographique compréhensive des mentalités croyantes contemporaines, faisant apparaître leurs implications en termes de constitution des réputations, formations des identités et cristallisation des conflits à l'échelle locale. Si la perspective de l'auteur demeure, en l'occurrence, un peu étroitement microscopique, elle correspond, au plan méthodologique, à une voie de recherche prometteuse.

- La question qui se pose ici - et qui ouvre un troisième niveau d'analyse de la sécularisation - est évidemment celui du devenir actuel de ce fonds chrétien, un et pluriel, confronté à une donne culturelle radicalement nouvelle, à des problèmes qui mettent en question les évidences éthiques, les constructions politiques et le rapport au monde qu'il a contribué à modeler pendant des siècles. Le problème est évidemment beaucoup plus complexe que celui de la reproduction institutionnelle des Églises chrétiennes que S. Bruce considère comme résolu (par évidement pur et simple) à l'horizon 2030. La question posée est celle des remaniements en cours et à venir de l'univers symbolique, moral, politique et juridique issu du christianisme, sous l'effet des mutations sociales, politiques, culturelles, scientifiques et techniques aujourd'hui en cours en Grande-Bretagne.

L'ouvrage de C.G. Brown se place déjà sur ce terrain en suggérant que la révolution récente des rapports entre hommes et femmes en Grande-Bretagne a constitué le facteur principal de l'ébranlement de ce fonds civilisationnel religieux qui a survécu durablement à l'affaissement de la religion institutionnelle. Le développement actuel des recherches sur les implications d'un multiculturalisme qui démultiplie les affirmations identitaires religieuses (non-chrétiennes) et bouleverse la problématique des relations entre l'Église et l'État héritée d'une longue histoire chrétienne contribue à ce changement de plan. Le problème est d'autant plus complexe que la question du «disestablishment» occupe autant actuellement les Églises chrétiennes libres (et plus généralement les Églises non anglicanes) que les minorités musulmanes et sikhs qui admettent jusqu'à un certain point la fonction de gestionnaire de la symbolique identitaire nationale dévolue à l'Église anglicane (tout en réclamant leur reconnaissance dans l'espace public). À ce propos, l'ouvrage collectif dirigé par T. Modood (1997) fait bien apparaître les conditions d'émergence de la question du désétablissement dans le débat public britannique. La discussion que suscite le problème - désormais officiellement posé - de la représentation des différentes Églises et confessions au sein de la Chambre des Lords cristallise, de façon particulièrement éclairante, ce remaniement des références partagées au sein de la société britannique. (Davie, 2000b). La question de la visibilité et de la reconnaissance (formelle et informelle) de la présence sociale et politique des minorités religieuses - musulmanes, hindouistes, bouddhistes etc. suscite un ensemble d'études qui réordonnent, à leur façon, la lecture classique de 
la sécularisation. Ce sont là des questions que les travaux de nos collègues James Beckford et Eileen Barker ont contribué depuis longtemps à mettre à jour et à formaliser. Tout récemment paru, l'ouvrage de M. Nye (2001) scrute avec perspicacité, à partir du cas de l'organisation ISKON, la situation ambiguë des groupes religieux controversés, dans un pays hautement sécularisé où existe une Église établie. Entendons bien qu'il ne s'agit pas seulement, dans la perspective introduite par ces travaux, d'analyser les formes actuelles du pluralisme religieux en Grande-Bretagne et d'étudier «à plat » les enjeux politiques et juridiques que la gestion des minorités religieuses dans l'espace public contribue à faire émerger. Le problème posé est aussi celui de la mutation culturelle qu'induit, comme telle, cette pluralisation concurrentielle des espaces symboliques et des univers axiologiques, dans une nation longuement modelée par le christianisme ${ }^{6}$.

On peut imaginer, plus avant, que la question des moyens nouveaux de communication (dans la ligne esquissée notamment par G. Davie, 2000, chap. 6) ou encore celle des changements qui affectent (à travers, par exemple, les performances de la médecine, le traitement des épizooties ou le développement du génie génétique) le rapport de la société britannique à la nature ouvrent à leur tour de nouveaux champs d'investigation pour une sociologie de la sécularisation, en invitant à scruter la façon dont les référents symboliques et éthiques issus du christianisme interviennent et se remanient éventuellement au fil du débat public sur ces questions. Il est douteux, si l'on récapitule l'ensemble (non limitatif !) de ces pistes de recherche dans lesquelles plusieurs de nos collègues britanniques sont engagés, que la question sociologique du devenir du christianisme en Grande-Bretagne puisse être considérée comme définitivement close !

Danièle HERVIEU-LÉGER

Centre d'Études Interdisciplinaires des Faits Religieux EHESS - Paris

${ }^{6}$ On se doit de signaler ici le livre récent de James BECKFORD (Religion in Prison. Equal Rights in a Multi-Faith Society, Cambridge-New York, Cambridge University Press, 1998, 232 p.) qui met en évidence, de façon précisément ciblée, les enjeux multiples (et les limites) de la reconnaissance du pluralisme religieux dans l'univers carcéral. Mais cet ouvrage fera l'objet, compte tenu des comparaisons que la recherche sur ce thème engage à l'échelle européenne, d'une présentation spécifique dans les pages des $A S S R$. 